\title{
NANO IMPRINT LITHOGRAPHY OF TEXTURES FOR LIGHT TRAPPING IN THIN FILM SILICON SOLAR CELLS
}

\author{
W. Soppe ${ }^{1}$, M. Dörenkämper ${ }^{1}$, J.B. Notta ${ }^{1}$, P.P.A.C. Pex ${ }^{1}$, W. Schipper ${ }^{2}$ and R. Wilde ${ }^{2}$ \\ 1) ECN-Solliance, High Tech Campus 5, 5656 AE Eindhoven, The Netherlands \\ Phone: +31 88515 4087, Fax: +31 88515 8214, E-mail: soppe@ecn.nl \\ 2) Nanoptics GmbH, Innungsstrasse 5, 21244 Buchholz, Germany
}

\begin{abstract}
Nano Imprint Lithography (NIL) is a versatile and commercially viable technology for fabrication of structures for light trapping in solar cells. We demonstrate the applicability of NIL in thin film silicon solar cells in substrate configuration, where NIL is used to fabricate a textured rear contact of the solar cells. We applied random structures, based on the natural texture of SnO:F grown by APCVD, and designed 2D periodic structures and show that for single junction $\mu \mathrm{c}-\mathrm{Si}$ cells these textured rear contacts lead to an increase of $\mathrm{J}_{\mathrm{sc}}$ of more than $40 \%$ in comparison to cells with flat rear contacts. Cells on optimized periodic textures showed higher fill factors which can be attributed to reduced microcrack formation, leading to less shunting in comparison to cells on random textures. Keywords: a-Si/ $\mu-\mathrm{Si}$, Light Trapping, Nano Imprint Lithography
\end{abstract}

\section{INTRODUCTION}

To boost cell efficiencies, while reducing materials costs, all thin film PV technologies seek ways to reduce the thickness of absorber layers. This, however, requires improved light management strategies even for strong light absorbing materials like CIGS. In order to tailor the light management for various cell concepts and optical properties of all cell components a flexible light management strategy is highly desired. UV NIL texturization offers this flexibility since virtually all imaginable textures can be made by this technology $[1,2,3]$.

ECN and Nanoptics are developing a novel fabrication process based on nano-imprint lithography (NIL) of textures for light trapping in thin film solar cells such as thin-film Si, OPV, CIGS and CdTe.

The process can be applied in roll-to-roll mode when using a foil substrate or in roll-to-plate mode when using a glass substrate. The UV lacquer also serves as an electrically insulating layer for cells if steel foil is used as substrate, to enable monolithic series interconnection.

\section{EXPERIMENTAL}

2.1 Nano Inprint Lithography (NIL)

NIL starts with designing or selection of the master structures that will be used in the replication process. Basically two routes can be followed in this: usage of a designed periodic structure, which is typically a result of optical modeling of light trapping or the usage of naturally grown random structures, which have proven to be good light scatterers, like the texture of SnO:F grown by APCVD or of $\mathrm{ZnO}: \mathrm{B}$ grown by LPCVD. In our research program we follow both routes. As a reference process we use masters originating from the texture of SnO:F, namely the Asahi-U type glass. In the competing process we use periodic 2-D sinusoidal structures with ideal periods and heights resulting from optical modeling [4]. These periodic master textures are typically made by interference lithography (IL). The dimensions of both type of masters is typically in the range of $10 \times 10 \mathrm{~cm}^{2}$. The next step is the fabrication of stamps based on the master structure. For smaller stamps, used in the ECN base line process, with substrate sizes up to $10 \times 10 \mathrm{~cm}^{2}$ we can make these stamps by 1:1 replication of the masters. For the larger scale imprinting, where larger stamps up to $100 \times 200 \mathrm{~cm}^{2}$ stamps are required, these stamps (called shims) are made by a step and repeat process.

\subsection{Optical properties}

We measured the diffuse and the total reflection of the textured back contact (stack configuration: steel foil/textured UV lacquer/Ag/ZnO) with an integrating sphere in the wavelength region from 350 to $1300 \mathrm{~nm}$.

\subsection{Solar cells}

The solar cells were processed according to the following scheme: 1) steel foil cleaning; 2) application of UV lacquer; 3) NIL; 4) Ag/ZnO back contact formation by magnetron sputtering; 5) deposition of silicon nip layer stack by RF-PECVD; 6) ITO front contact formation by magnetron sputtering; 7) evaporation of Ag grid contacts; 8) annealing. We made single junction $\mu \mathrm{c}-$ Si cells and varied the thickness of the $\mu \mathrm{c}-\mathrm{Si}$ absorber layer: 500, 750 and $1000 \mathrm{~nm}$.

After annealing the $V_{o c}$ and $F F$ of the cells were determined by IV measurements in a solar simulator. Jsc of the cells was determined by spectral response measurements at $0 \mathrm{~V}$ and no bias light.

\section{RESULTS}

\subsection{Replication quality and Si layer growth}

The replication method that we have developed appears to provide excellent replication quality. For the periodic textures, the period and height of the replicated structures are - within error margins - identical to that of the original. For the random texture the RMS roughness as determined by Atomic Force Microscopy (AFM) is likewise identical for master, stamp and replication in UV lacquer.

In Figure 2 a SEM image of the replication of the periodic texture in UV lacquer, coated with a $\mathrm{Ag} / \mathrm{ZnO}$ back contact is shown. The thickness of the back contact is respectively 200 and $80 \mathrm{~nm}$ for the $\mathrm{Ag}$ and the $\mathrm{ZnO}$ layer and it can be observed that the back contact forms a conformal coating on the periodic structure. 


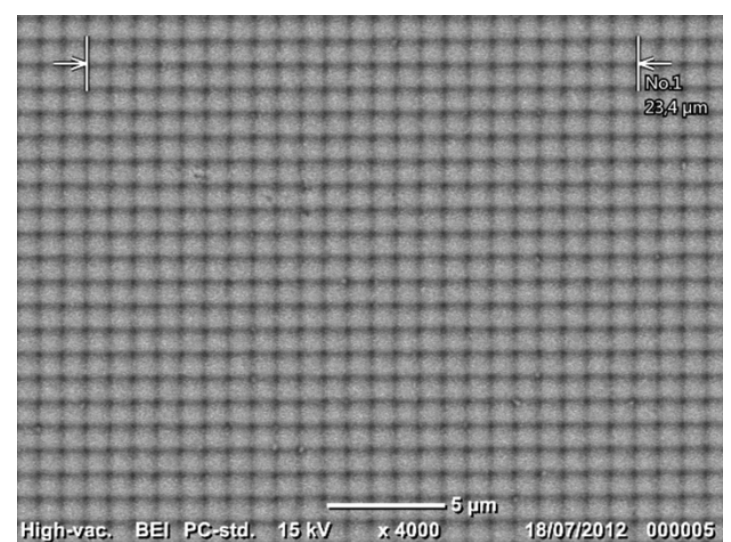

Figure 1: SEM image of a replica of the periodic structure in lacquer on steel foil. The structure is covered by a $\mathrm{Ag} / \mathrm{ZnO}$ back contact

This conformal growth is confirmed by cross sectional SEM images of the entire cells. In Figure 2 we show a typical example; in this case a $\mu c-S i$ cell with a thickness of $1000 \mathrm{~nm}$. It is interesting to see that - for this layer thickness - the conformal growth finally leads to a reversed periodic structure at the top of the cell.

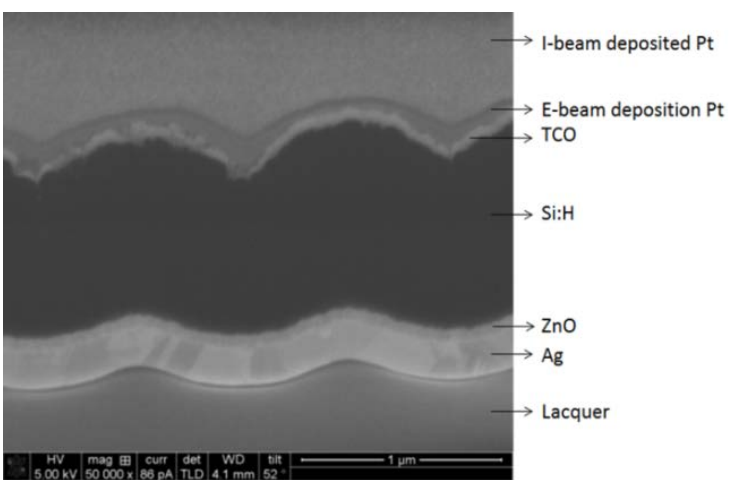

Figure 2: Cross sectional SEM image of a single junction $\mu \mathrm{c}-\mathrm{Si}$ cell on periodic texture.

\subsection{Light scattering properties}

The haze values of the reflection of the random and periodic textured back contacts are shown in Figure 3. It can be observed that the haze factor of the periodic texture is appreciably higher than that of the random texture in the crucial wavelength region from 700 to 1000 $\mathrm{nm}$. But one has to be cautious to draw quick conclusions from this since the haze factor is only a limited predictor of the light trapping capability. For more accurate predictions of the light trapping, angular distribution functions of the reflection are required and we hope to be able to present these too in near future.

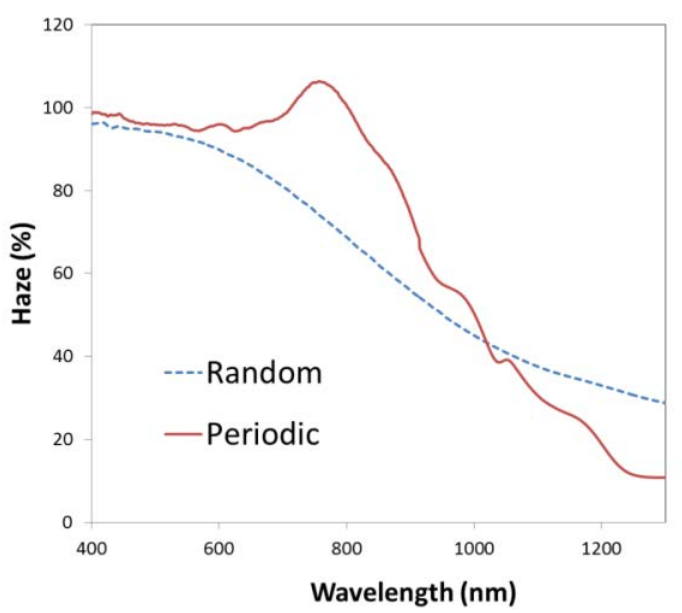

Figure 3: Haze values for the reflection of the random and the periodic textured back contacts.

\section{$3.3 \mu \mathrm{c}-\mathrm{Si}$ cells}

We made larger series of nip $\mu \mathrm{c}$-Si cells with areas of $4 \times 4 \mathrm{~mm} 2$ and $10 \times 10 \mathrm{~mm} 2$, on flat substrates, on substrates with a random texture (replica of Asahi-U type) and substrates with a periodic texture. In general the cells on the flat substrate had the highest Voc (typically $530 \mathrm{mV}$ ) and FF (typically 70\%), whereas the cells on periodic textures showed similar FFs, but smaller $\mathrm{V}_{\text {oc }} \mathrm{S}$ (typically $500-510 \mathrm{mV}$ ). The cells on random textures had both lower $\mathrm{V}_{\mathrm{oc}} \mathrm{S}$ (in the same range as for the cells on periodic textures) but also lower FFs (in the range of $65 \%$ ). We attribute the lower $\mathrm{V}_{\text {oc }}$ values to the increase of the junction area due to the texture, leading to larger dark currents. The decrease in FF is clearly related to the observed decreased $\mathrm{R}_{\text {shunt}}$; which is probably due to an increased number of shunting paths in cells with a sharply textured back contact.

The light trapping effect of the textured back contact can be clearly observed when comparing the $\mathrm{J}_{\mathrm{sc}}$ of the cells. Highest values of more than $24 \mathrm{~mA} / \mathrm{cm}^{2}$ were obtained for the cells on periodic textures (See Figure 4). The increase of $\mathrm{J}_{\mathrm{sc}}$ with respect to a flat back contact is $41 \%$ ! This increase is even larger than predicted by optoelectrical modeling [4].

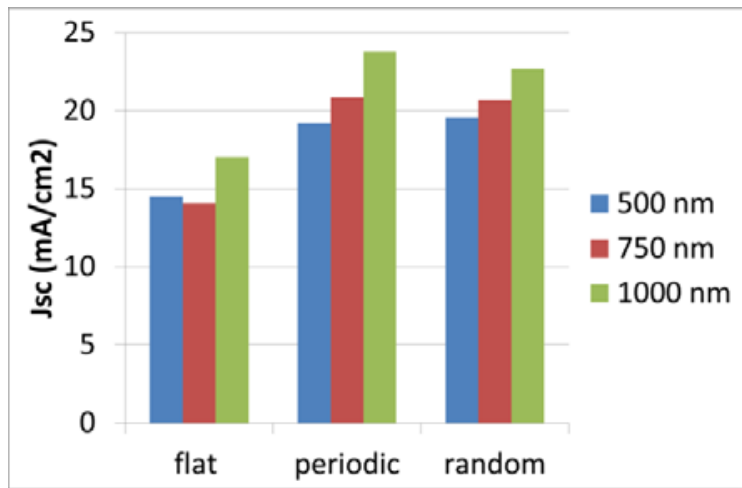

Figure 4: $\mathrm{J}_{\mathrm{sc}}$ of single junction $\mu \mathrm{c}-\mathrm{Si}$ cells with various absorber thicknesses on periodic and on random textures.

The spectral response of cells with an absorber layer thickness of $750 \mathrm{~nm}$ is shown in Figure 5 . The gain of $\mathrm{J}_{\mathrm{sc}}$ resulting from the texture at the rear side is roughly the same in the long wavelength regime, for the periodic and the random texture. It is noteworthy that the periodic 
texture leads to a higher response in the blue part of the spectrum than the random texture. This might be due to lower losses in the $\mathrm{p} / \mathrm{ITO}$ layers with the resulting type of morphology of the top layers.

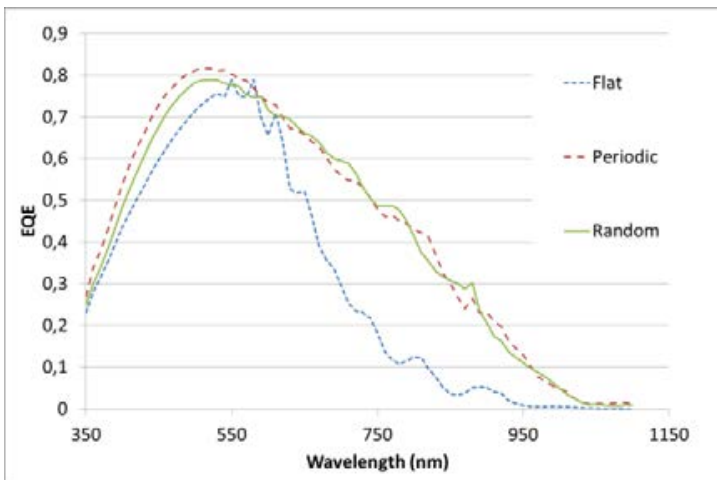

Figure 5: Spectral response data of cells made with an absorber layer thickness of $750 \mathrm{~nm}$.

\section{DISCUSSION AND OUTLOOK}

Nano imprint lithography is a versatile tool to fabricate light scattering structures in solar cells. Since scaling up to large areas is also well possible, we foresee industrial implementation on a relatively short term. In this paper we have shown that NIL can be well applied for fabricating of light scattering textures at the rear side of thin film silicon solar cells. With the periodic sinusoidal structure we obtained a $J_{\mathrm{sc}}$ of $24 \mathrm{~mA} / \mathrm{cm}^{2}$, an increase of more than $40 \%$ in comparison with a flat back reflector.

\section{ACKNOWLEDGEMENTS}

This work was funded by the European FP7 project

Silicon-Light (GA No. 241277)

\section{REFERENCES}

[1] H. Hauser,et al, Energy Procedia 8, 648-653 (2011).

[2] C. Bataglia et al., APL 96, 213504, (2010).

[3] K. Söderström et al., PIP 19, 202-210 (2010)

[4] B. Lipovšek, M. Cvek, A. Čampa, J. Krč, M. Topič, 25th European Photovoltaic Solar Energy Conference (2010) 